\title{
Inventory of the volatiles on comet 67P/Churyumov-Gerasimenko from Rosetta/ROSINA
}

\author{
Léna Le Roy ${ }^{1, \star}$, Kathrin Altwegg ${ }^{1,2}$, Hans Balsiger $^{2}$, Jean-Jacques Berthelier ${ }^{3}$, Andre Bieler $^{4}$, Christelle Briois ${ }^{5}$, \\ Ursina Calmonte ${ }^{2}$, Michael R. Combi ${ }^{4}$, Johan De Keyser ${ }^{6}$, Frederik Dhooghe ${ }^{6}$, Björn Fiethe ${ }^{7}$, Stephen A. Fuselier ${ }^{8,9}$, \\ Sébastien $\mathrm{Gasc}^{2}$, Tamas I. Gombosi ${ }^{4}$, Myrtha Hässig8, ${ }^{8,2}$ Annette Jäckel ${ }^{2}$, Martin Rubin², and Chia-Yu Tzou ${ }^{2}$ \\ 1 Center for Space and Habitability, University of Bern, 3012 Bern, Switzerland \\ e-mail: lena.leroy@csh.unibe.ch \\ 2 Physikalisches Institut, University of Bern, 3012 Bern, Switzerland \\ 3 LATMOS/IPSL-CNRS-UPMC-UVSQ, 4 avenue de Neptune, 94100 Saint-Maur, France \\ ${ }^{4}$ Department of Atmospheric, Oceanic and Space Science, University of Michigan, Ann Arbor, MI, USA \\ 5 Laboratoire de Physique et Chimie de l'Environnement et de l'Espace, LPC2E, CNRS 7328/Université d'Orléans, \\ $3 \mathrm{~A}$ av. de la Recherche Scientifique, 45071 Orléans, France \\ ${ }^{6}$ BIRA-IASB, Belgian Institute for Space Aeronomy, Ringlaan 3, 1180 Brussels, Belgium \\ 7 Institute of Computer and Network Engineering (IDA), TU Braunschweig, Hans-Sommer-Straße 66, 38106 Braunschweig, \\ Germany \\ 8 Space Science Division, Southwest Research Institute, 6220 Culebra Rd., San Antonio, TX 78238, USA \\ 9 Department of Physics and Astronomy, University of Texas at San Antonio, San Antonio, TX 78238, USA
}

Received 1 May 2015 / Accepted 30 July 2015

\section{ABSTRACT}

\begin{abstract}
Context. The ESA Rosetta spacecraft (S/C) is tracking comet $67 \mathrm{P} /$ Churyumov-Gerasimenko in close vicinity. This prolonged encounter enables studying the evolution of the volatile coma composition.

Aims. Our work aims at comparing the diversity of the coma of 67P/Churyumov-Gerasimenko at large heliocentric distance to study the evolution of the comet during its passage around the Sun and at trying to classify it relative to other comets.

Methods. We used the Double Focussing Mass Spectrometer (DFMS) of the ROSINA experiment on ESA's Rosetta mission to determine relative abundances of major and minor volatile species. This study is restricted to species that have previously been detected elsewhere.

Results. We detect almost all species currently known to be present in cometary coma with ROSINA DFMS. As DFMS measured the composition locally, we cannot derive a global abundance, but we compare measurements from the summer and the winter hemisphere with known abundances from other comets. Differences between relative abundances between summer and winter hemispheres are large, which points to a possible evolution of the cometary surface. This comet appears to be very rich in $\mathrm{CO}_{2}$ and ethane. Heavy oxygenated compounds such as ethylene glycol are underabundant at $3 \mathrm{AU}$, probably due to their high sublimation temperatures, but nevertheless, their presence proves that Kuiper belt comets also contain complex organic molecules.
\end{abstract}

Key words. comets: individual: 67P/Churyumov-Gerasimenko

\section{Introduction}

Comets are visitors from the outer solar system. They are also important witnesses to the formation of our solar system. Because of their small size and the fact that they have spent 4.5 billion years far from the Sun where temperatures are low, comets are understood to be the least processed bodies in the solar system and thus represent time capsules with material from the early stages of our solar system. However, the chemical composition of comets could have changed through thermal and physical processes that they were subjected to until today. It is therefore important to measure the volatile inventory of chemical species in comets and compare it to other bodies in the solar system, interstellar matter, and protostellar objects. This comparison tells us about the primordial material and about

* Present address: Center for Space and Habitability, University of Bern, Sidlerstrasse 5, 3012 Bern, Switzerland. the formation processes, and it permits assessing the exogenous delivery of water, carbon, and prebiotic molecules to the early Earth.

Remote-sensing observations have improved our knowledge of a comet's volatile composition. A number of species are detected with ground-based and near-Earth space-based telescopes in the visual, infrared, microwave, and radio portions of the spectrum. A limitation is that symmetric species cannot be measured with radio or submillimeter observations, and that measurement from space is appropriate for species present in Earth's atmosphere (e.g., $\mathrm{CO}_{2}$ ). Such observations have resulted in the identification of a number of volatile species for very many comets. The most extensive list of identified molecules up to now has been established from observations of $\mathrm{C} / 1995$ O1 (Hale-Bopp) and C/1996 B2 (Hyakutake) (Bockelée-Morvan et al. 2004; Mumma \& Charnley 2011). Compilations of volatile compositions are often dominated by data from comets that are 
Table 1. Fragmentation pattern and sensitivity used to calculate the relative abundance.

\begin{tabular}{ccc}
\hline \hline Species & Fragmentation pattern & Sensitivity \\
\hline $\mathrm{H}_{2} \mathrm{O}$ & $\mathrm{M}$ & $\mathrm{M}$ \\
$\mathrm{CO}$ & $\mathrm{NIST}$ & $\mathrm{C}$ \\
$\mathrm{CO}_{2}$ & $\mathrm{M}$ & $\mathrm{M}$ \\
$\mathrm{CH}_{4}$ & $\mathrm{M}$ & $\mathrm{M}$ \\
$\mathrm{C}_{2} \mathrm{H}_{2}$ & $\mathrm{NIST}$ & $\mathrm{C}$ \\
$\mathrm{C}_{2} \mathrm{H}_{6}$ & $\mathrm{M}$ & $\mathrm{M}$ \\
$\mathrm{CH}_{3} \mathrm{OH}$ & $\mathrm{NIST}$ & $\mathrm{C}$ \\
$\mathrm{H}_{2} \mathrm{CO}$ & $\mathrm{NIST}$ & $\mathrm{C}$ \\
$\mathrm{HCOOH}_{\mathrm{CH}}$ & NIST & $\mathrm{C}$ \\
$\mathrm{OHCH}_{2} \mathrm{OH}$ & NIST & $\mathrm{C}$ \\
$\mathrm{HCOCH}_{3}$ & NIST & $\mathrm{C}$ \\
$\mathrm{CH}_{3} \mathrm{CHO}$ & NIST & $\mathrm{C}$ \\
$\mathrm{NH}_{3}$ & $\mathrm{M}$ & $\mathrm{M}$ \\
$\mathrm{HCN}$ & NIST & $\mathrm{C}$ \\
$\mathrm{HNCO}_{\mathrm{CH}_{3} \mathrm{CN}}$ & Fischer et al. (2002) & $\mathrm{C}$ \\
$\mathrm{H}_{2} \mathrm{~S}$ & NIST & $\mathrm{C}$ \\
$\mathrm{OCS}^{\mathrm{SO}}$ & $\mathrm{M}$ & $\mathrm{M}$ \\
$\mathrm{SO}_{2}$ & NIST & $\mathrm{C}$ \\
$\mathrm{CS}_{2}$ & estimated & $\mathrm{C}$ \\
$\mathrm{S}_{2}$ & NIST & $\mathrm{C}$ \\
& NIST & $\mathrm{C}$ \\
& estimated & $\mathrm{C}$ \\
\hline
\end{tabular}

Notes. $\mathrm{M}$ refers to fragmentation pattern and sensitivity determined in the calibration facility. $\mathrm{C}$ refers to the sensitivities that were calculated from the function determined thanks to the calibration measurements.

understood to come from the Oort Cloud reservoir, the longperiod comets (LPCs), and the intermediate-period Halley-type comets (HTCs) that are bright enough to be observed by IR and radio; Jupiter-family comets (JFCs), with fainter gas and dust production, have only rarely been investigated by radio and IR observations (Crovisier et al. 2009).

1P/Halley is the first HTC to date for which there exists a detailed volatile composition measurement, obtained in situ by mass spectrometry during the flyby of the European Space Agency's Giotto spacecraft in 1986 (Eberhardt 1999), recently complemented with remote-sensing observations (Rubin et al. 2011). We list in Tables 2 to 5 some molecules for a number of comets that we expanded with data collected from space missions to comets 1P/Halley, 9P/Tempel 1, and 103P/Hartley 2.

Taxonomic classification of comets is based (in part) on their volatile composition by identifying groups of comets that have a typical composition or that are depleted or enriched compared with the typical composition. For a detailed and comprehensive summary on chemical composition of comets based on radio and IR observations, see Mumma \& Charnley (2011). More systematic taxonomic classifications of comets have been done in surveys of visible-range remote observations of comets that cover a number of radical species $\left(\mathrm{C}_{2}, \mathrm{C}_{3}, \mathrm{CN}, \mathrm{NH}, \mathrm{NH}_{2}, \mathrm{OH}\right.$, $\left.\mathrm{O}\left({ }^{1} \mathrm{D}\right)\right)$, and the dust continuum measured as $\operatorname{Af} \rho$ ). Several surveys based on photometric or spectrophotometric observations of comets have been carried out over the past $30 \mathrm{yr}$ (Cochran et al. 2012, 130 comets; A'Hearn et al. 1995, 85 comets; Fink 2009, 50 comets; Langland-Shula \& Smith 2011, 26 comets).

There are a few difficulties in comparing ground-based visual radical taxonomies with abundances of molecules observed in the IR and radio. First, the ground-based visual observations cover many more comets, and most of these comets are much fainter than the comets that are bright enough to be observed in the IR and radio. Therefore, the visual-range surveys are dominated by observations of short-period JFCs, while the IR and radio surveys are dominated by LPCs or new Oort Cloud comets.

Second, despite decades of studies trying to clearly identify the parent molecules of the common cometary radicals as well as the chains of branching ratios and production or destruction rates needed to calculate overall production rates, there are only few hard and fast connections. Generally, it is understood that $\mathrm{NH}$ and $\mathrm{NH}_{2}$ radicals come from $\mathrm{NH}_{3}$ photodissociation (Fink et al. 1991). $\mathrm{HCN}$ is probably the main parent of $\mathrm{CN}$, but various attempts to demonstrate the detailed quantitative connection that can easily be established (Combi \& Delsemme 1980; Woodney et al. 2002; Fray et al. 2005) seem to work sometimes, but not always. A similar case has been made to connect $\mathrm{C}_{2}$ to parents such as $\mathrm{C}_{2} \mathrm{H}_{2}$ and $\mathrm{C}_{2} \mathrm{H}_{6}$ (Helbert et al. 2005) with similar mixed success. More recently, Weiler (2012) studied the chemistry of $\mathrm{C}_{2}$ and $\mathrm{C}_{3}$ in cometary comae and concluded that $\mathrm{C}_{2} \mathrm{H}_{6}$ has little influence on $\mathrm{C}_{2}$. Combi \& Fink (1997) have made a case from analyzing its spatial distribution that $\mathrm{C}_{2}$ is either mainly emitted as a granddaughter product of one or more molecules or perhaps emitted from organic grains.

In Tables 2 to 5 we show a list of parent molecule chemical compositions in a number of comets, not only including bright Oort Cloud Comets (1P/Halley, C/1995 O1 (Hale-Bopp), C/1996 B2 (Hyakutake), C/2001 A2 (LINEAR), C/2012 F6 (Lemmon), and C/2013 R1 (Lovejoy)), but also including more recent results from infrared observations of a few short-period JFCs (103P/Hartley 2, broken 73P/SchwassmannWachmann 3 (73P/SW3) fragments $\mathrm{B}$ and $\mathrm{C}, 2 \mathrm{P} /$ Encke, 9P/Tempel 1 before and after Deep Impact excavation, 6P/d'Arrest, 17P/Holmes, and 21P/Giacobini-Zinner). Tables 2 to 5 also list the classifications of each comet according to ground-based visual observations (A'Hearn et al. 1995; Fink 2009; Cochran et al. 2012; Langland-Shula \& Smith 2011). These classifications generally agree with one another, although Fink (2009) has refined some extra classification that places comets 9P/Tempel 1 and 67P/Churyumov-Gerasimenko (hereafter $67 \mathrm{P}$ ) in the "typical" subclass but for which $\mathrm{C}_{2}$ is somewhat depleted.

The aim of this paper is to present high-resolution measurements from the Double Focusing Mass Spectrometer (DFMS), part of the Rosetta Orbiter Sensor for Ion and Neutral Analysis (ROSINA) of the inventory of cometary volatiles during two snapshot periods in the coma of 67P. Here we limit the analysis to molecules that have previously been detected in one or several other comets. We report their abundances relative to water. It is the first time that such an abundance list, including sulfur-, nitrogen-, and oxygen-bearing compounds, is provided for a JFC. Section 2 presents the conditions during the two observations. In Sect. 3 the principle of DFMS as well as the data treatment to retrieve the parent species and their abundances are described. Section 4 focuses on the abundances measured in the coma of $67 \mathrm{P}$ and contains its classification.

\section{Observations}

The measurements presented in this paper have been obtained before lander delivery. The data were acquired on 20 October 2014 between 07:54 and 08:37 (UTC) and on 19 October 2014 between 00:39 and 01:22 (UTC). The analyzed mass spectra with integration times of 20 s were not averaged. Therefore the 
L. Le Roy et al.: Volatile inventory of 67P/Churyumov-Gerasimenko

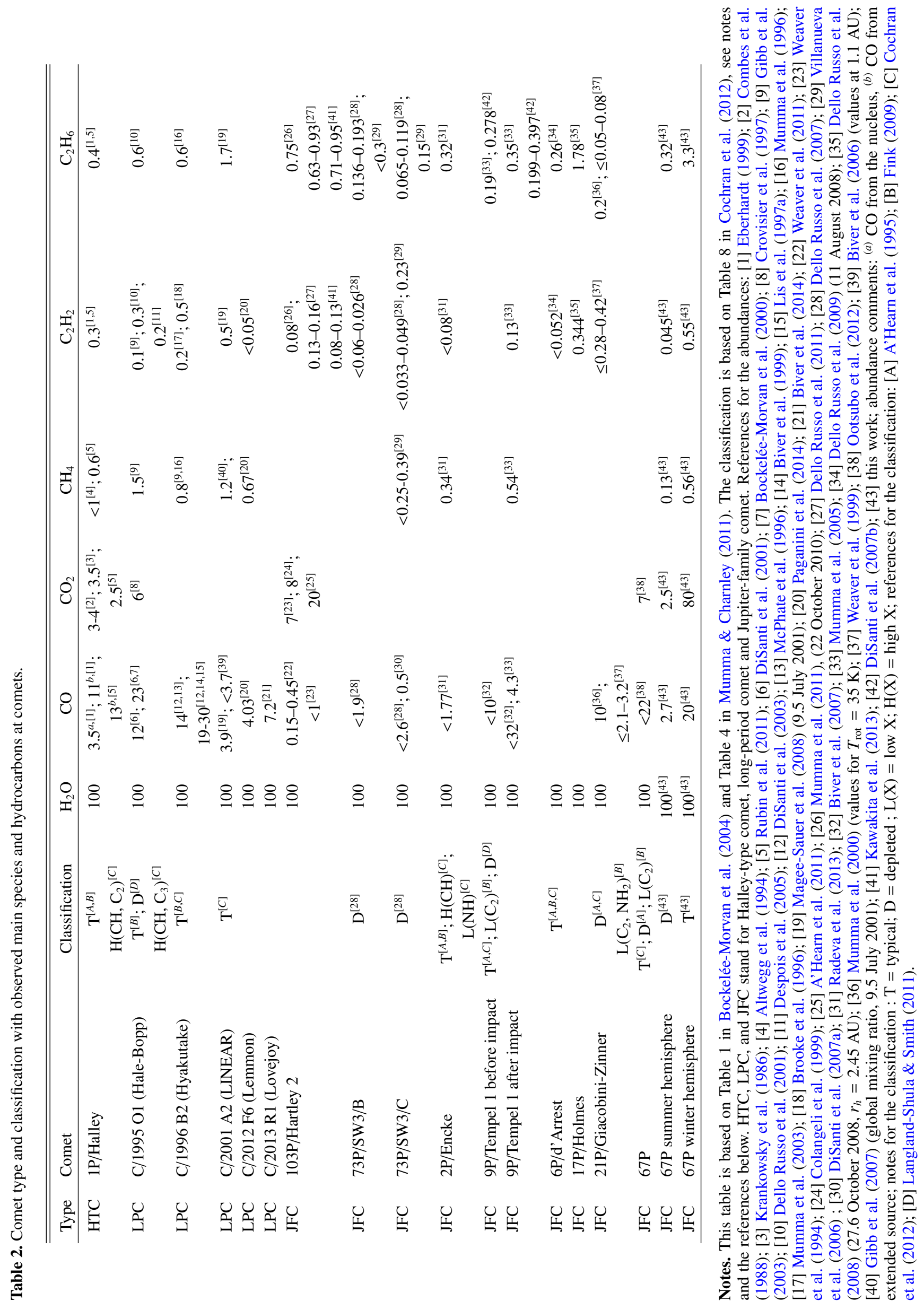




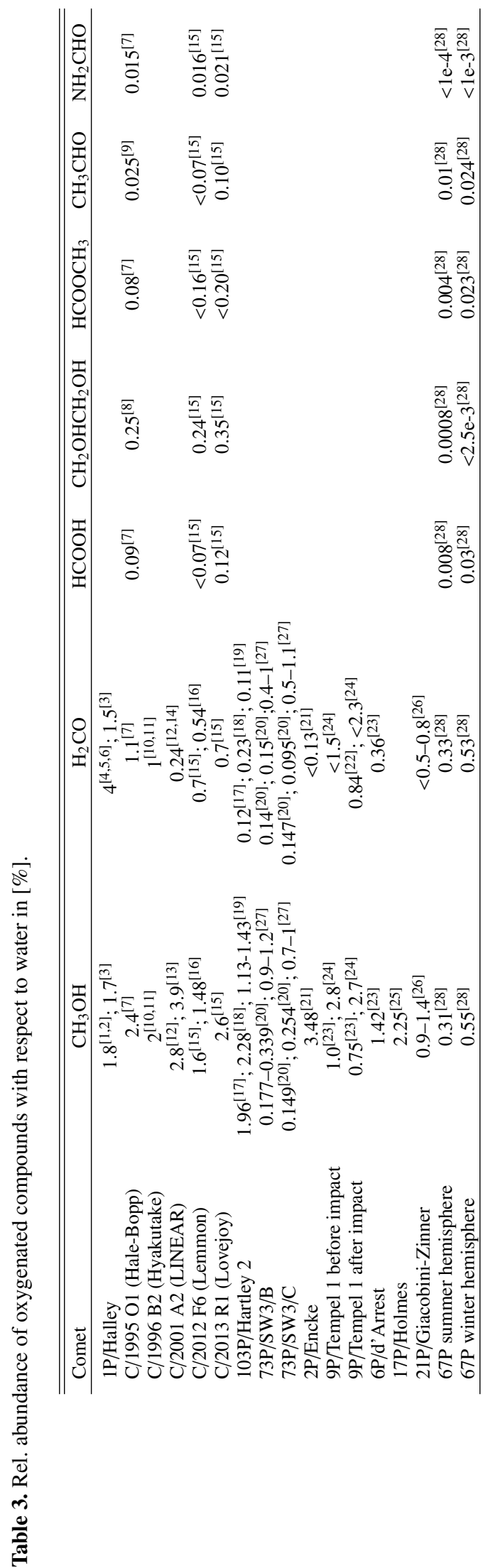

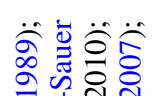

$\smile$ i่

屯

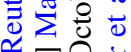

\& $\stackrel{0}{0}$.

药

贯它吉

兄过.

후 त

$\ddot{0} \approx 0 त$

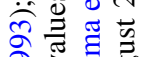

。류.

요요

엉

牙

䨌

๘.

的秃

$\infty$ 등

可

苓交

次芯䨌

ठ드을

휴 च르

正云守官

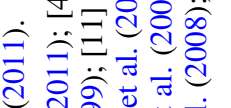

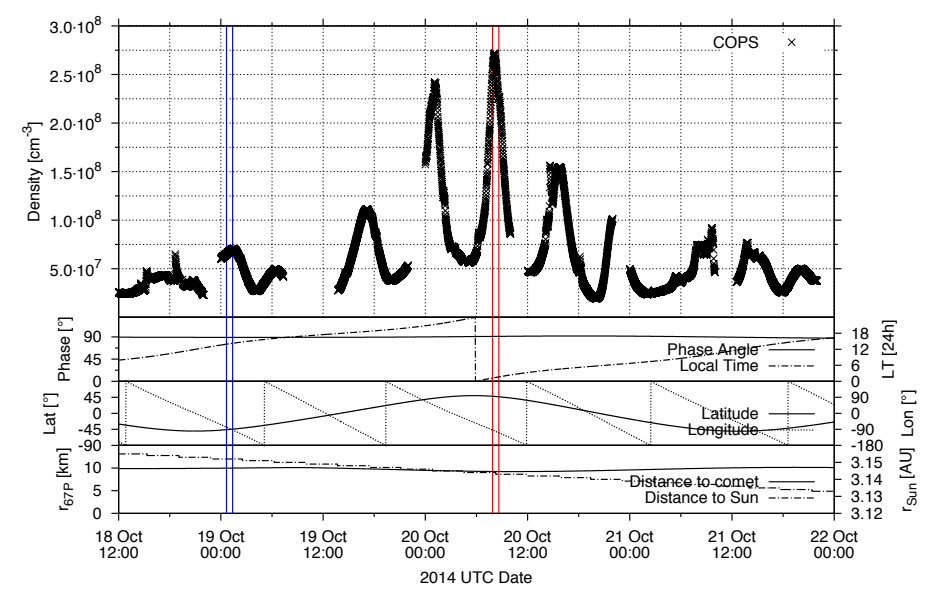

(a) COPS density and S/C location

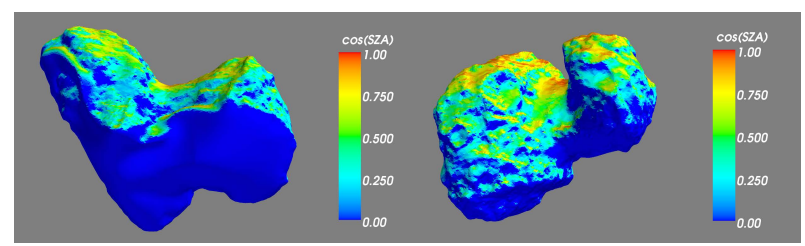

(b) 19 October 2014

(c) 20 October 2014

Fig. 1. a) COPS data, spacecraft footpoint, and comet position for the period between 18-21 October, 2014 (noon till midnight). The bottom panel shows the distance to the nucleus center, $r_{67 \mathrm{P}}$, and the heliocentric distance, $r_{\text {Sun }}$. The second panel from the bottom shows latitude and longitude, and above are phase angle and local time, LT. The top panel shows the measured COPS densities. The two reported intervals are indicated in blue (winter hemisphere at negative latitude, 19 October 2014 00:39:25-01:22:18) and red (summer hemisphere at positive latitude, 20 October 2014 07:53:51-08:37:45). Both observations occur at approximately the same longitude and at a phase angle of $90^{\circ}$. b) and c) show the illumination conditions as seen from the Rosetta spacecraft during the observations above the winter hemisphere on 19 October 2014 and above the summer hemisphere on 20 October 2014.

results presented are snapshots of the coma composition at the location of the spacecraft during the indicated times.

At these dates, Rosetta was on a terminator orbit with a period of $2 \mathrm{~d} 17 \mathrm{~h}$, at $10 \mathrm{~km}$ from the nucleus. The different conditions are shown in Fig. 1. The upper panel shows the geometrical information for the $\mathrm{S} / \mathrm{C}$ position at that time together with the measured total density from the COmet Pressure Sensor (COPS), part of the ROSINA experiment. COPS shows the diurnal and seasonal variations of the comet atmosphere as described by Bieler et al. (2015). The spectra are shown for the two observation intervals: one was measured over negative latitudes (blue), which corresponds to the winter hemisphere, the other one (red) over positive latitudes, which corresponds to summer. COPS data clearly show that outgassing is much higher over the summer hemisphere. This outgassing is mostly water. Other molecules, for example, $\mathrm{CO}_{2}$, do not follow this trend. Their density can be higher over the winter hemisphere (Luspay-Kuti et al. 2015; Hässig et al. 2015). It is therefore clear that densities relative to water can vary over a wide range between the two hemispheres. It is currently not clear which values best represent the bulk composition of the nucleus. This will require detailed investigation of both hemispheres during 
perihelion when outgassing is highest, and winter and summer are reversed compared to the measurement period analyzed here.

\section{DFMS data treatment}

The results presented in this paper come mainly from the DFMS instrument (see Balsiger et al. 2007 for a detailed description). More precisely, they are based on the high-resolution mode observations.

\subsection{DFMS operating principle}

During the time of observation, the instrument was pointed in the nadir direction at a cometocentric distance of about $10 \mathrm{~km}$ from the center of mass, so that the neutral gas escaping from the comet nucleus was able to freely enter the instrument. Figure 2 shows a sketch of the ion optical elements of DFMS. A repelling electrostatic potential prevents the entry of positively charged ions. The principle of neutral detection and the data treatment process for the high-resolution mode are described here in detail.

The neutrals are bombarded by electrons that are emitted from a filament and accelerated through a $45 \mathrm{~V}$ potential in the ion source. The resulting ions (atomic or molecular ions or charged molecular fragments of the original neutrals) are then accelerated by the mass-dependent acceleration voltage $V_{\text {accel }}$. The analyzer section of DFMS has a Nier-Johnson geometry in which a deflection of $90^{\circ}$ in a radial electrostatic energy analyzer (ESA) is combined with a magnetic deflection of $60^{\circ} . V_{\text {accel }}$ determines which ions will be transmitted through the DFMS ion optics. The ESA focuses ions with different entry angles but with the same energy, while ions with different energies are spatially separated. The kinetic energy of the ions in the ESA is related to $V_{\text {accel }}$ and the ESA radius according to

$E_{\mathrm{ESA}}=\frac{2 V_{\mathrm{accel}}}{r_{\mathrm{ESA}}}$.

An energy slit after the ESA filters the ion energy to the desired value $\left(E_{\mathrm{ESA}} \pm 1 \%\right)$. Depending on $V_{\text {accel }}$ and the properties of the magnetic sector (magnetic field and magnetic sector radius, Eq. (2)), only ions centered around a certain m/q value, the commanded mass-over-charge (CM), will hit the detector as dictated by

$m / q=\frac{r_{B}^{2} B^{2}}{2 V_{\text {accel }}}$.

For heavy ions $(m / q>70)$, for which $V_{\text {accel }}$ is low, a postacceleration voltage is applied on the front side of the detector to increase the sensitivity. The Micro Channel Plate/Linear Electron Detector Array (MCP/LEDA) detector consists of two microchannel plates with angled channels rotated $90^{\circ}$ relative to each other that produce a chevron shape, placed in front of a position-sensitive imaging detector with two rows of 512 pixels (LEDA channels A and B). A detailed discussion of the detector setup and the MCP can be found in (Berthelier et al. 2002; Nevejans et al. 2002; Balsiger et al. 2007). Upon impact of an ion on the MCP, a cascade of electrons is produced, depending on the ion species, the energy of the ion, and the MCP performance. The overall MCP gain at the location of impact (possibly including some nonlinearity) is determined by selecting a gain step from a predefined list of steps, each of which corresponds to a setting of the potential difference between the MCP front and back planes. The LEDA analog measurements are converted into a digital signal and are obtained as ADC counts as a function of LEDA pixel number for both LEDA rows. Typically, 3000 individual $6.6 \mathrm{~ms}$ exposures at the same CM, equivalent to a $19.8 \mathrm{~s}$ total measurement time, are accumulated to produce a spectrum.

\subsection{Intensity correction}

The ADC counts for each spectrum and each LEDA channel need to be corrected for the offset that is due to the initial charge applied for proper operation. The offset function is fitted by removing regions where peak signals are detected and fitting the remaining ADC counts as a function of pixel number. The number of offset-corrected ADC counts (representing the number of electrons collected by each pixel) is divided by the overall MCP gain factor $\mathrm{g}_{\mathrm{MCP}}$ that corresponds to the selected gain step to obtain the number of ions that impinged on the MCP during the exposure.

The gain at each pixel of the LEDA detector arrays is a measure of the combined response of the MCP and the LEDA. This MCP/LEDA pixel gain, $g_{\mathrm{p}}$, evolves with time, mainly because impinging ions degrade the coating inside the MCP microchannels that is responsible for the electron cascade effect. Because of the choice of $\mathrm{CM}$ values, typically significant ion peaks are recorded near the middle of the pixel arrays; these center pixels therefore degrade faster than the other pixels. Consequently, detector aging needs to be checked on a regular basis. This aging effect can be compensated for by applying pixel gain correction factors. These represent the efficiency of the electron cascade process for each MCP/LEDA pixel for a certain detector gain step. They are obtained by collecting raw data for a certain ion (e.g., $\mathrm{H}_{2} \mathrm{O}^{+}$) in low-resolution mode for a sequence of $\mathrm{CM}$ values to scan the peak across the pixel arrays of the MCP/LEDA. Such pixel gain measurements are performed regularly every few months depending on the total accumulated charge on the detector. The dimensionless pixel gain correction factors are then calculated by taking the sum of the offset-corrected peak intensities for each pixel and scaling these such that $g_{\mathrm{p}}=1$ away from the center of the arrays; in doing so, the assumption is made that the pixels away from the center are aging less rapidly.

The ADC counts for each pixel are converted into ion counts per second using

$I(p)=\frac{\mathrm{ADC}}{g_{\mathrm{p}} g_{\mathrm{MCP}}} \frac{c_{\mathrm{adc}} c_{\text {leda }}}{t}$,

where $I(p)$ is the ion current per pixel, $c_{\text {adc }}=2.5 \mathrm{~V} /\left(2^{12}-1\right)$ is the ADC conversion factor, $\mathrm{c}_{\text {leda }}=4.2 \times 10^{-12} \mathrm{~F}$ is the LEDA capacitance, and the total integration time. The number of ions per second and per pixel can then be calculated by dividing $I(p)$ by the elementary charge e.

\subsection{Mass calibration}

Mass calibration consists of associating pixel numbers with mass-per-charge $(\mathrm{m} / \mathrm{q})$ ratios. The relation is given by

$M(p)=M_{0} e^{\left(p-p_{0}\right) \frac{x}{D z}}$,

where $M(p)$ gives the mass-per-charge ratio corresponding to pixel $p, M_{0}$ is the CM value, $p_{0}$ is the center MCP pixel where $M_{0}$ is located, $x=25 \mu \mathrm{m}$ is the center-to-center distance between adjacent pixels, $D=127000 \mu \mathrm{m}$ is the dispersion factor, and $z=6.4$ (somewhat smaller for lower masses) denotes the zoom factor associated with the high-resolution modes. 
A\&A 583, A1 (2015)

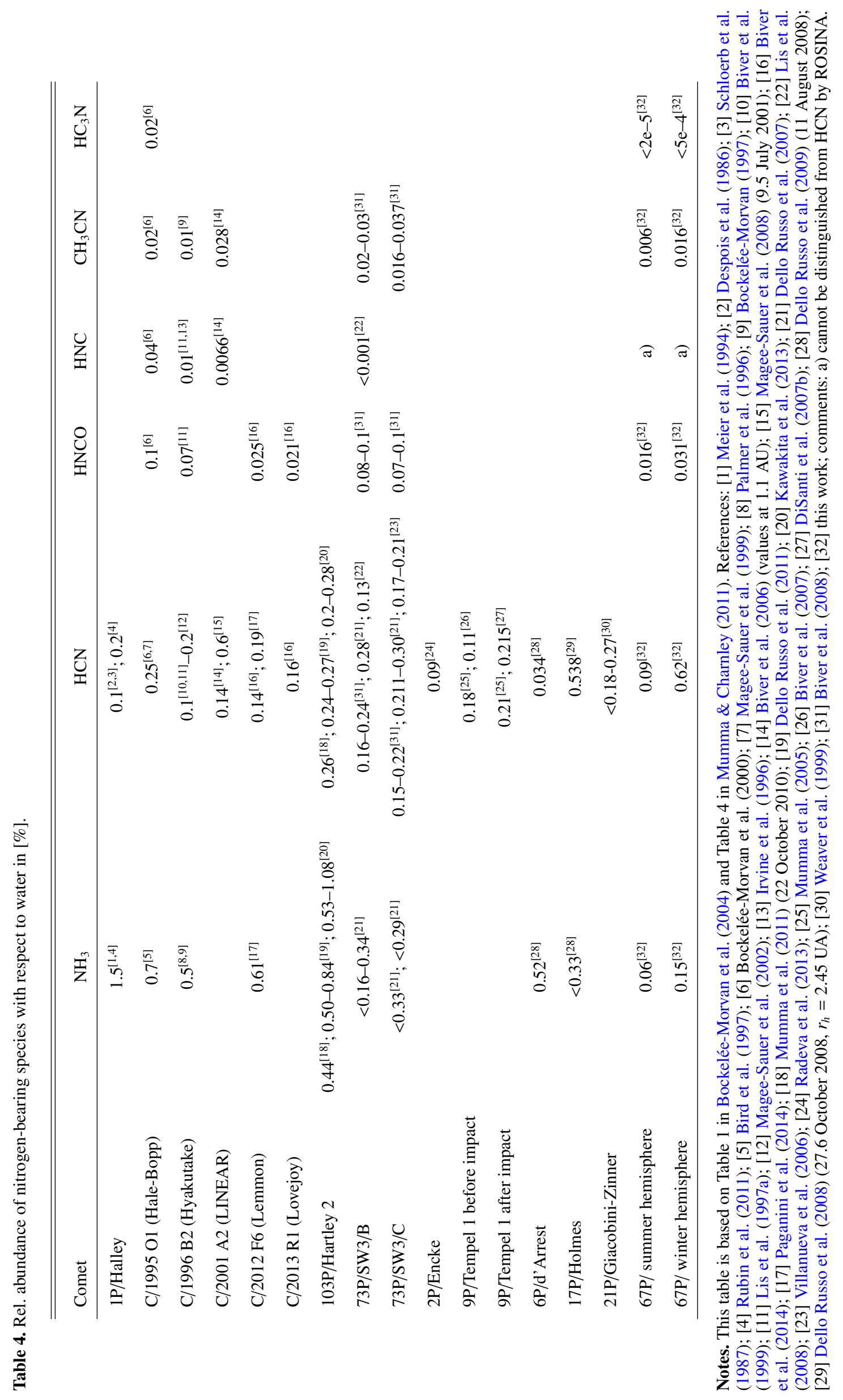


Table 5. Rel. abundance of sulfur-bearing species with respect to water in [\%].

\begin{tabular}{lccccccc}
\hline \hline Comet & $\mathrm{H}_{2} \mathrm{~S}$ & $\mathrm{OCS}$ & $\mathrm{SO}$ & $\mathrm{SO}_{2}$ & $\mathrm{CS}$ & $\mathrm{CS}_{2}$ & $\mathrm{~S}_{2}$ \\
\hline 1P/Halley & $0.4^{[1]}$ & & & & & $0.2^{[2]}$ & \\
C/1995 O1 (Hale-Bopp) & $1.5^{[3]}$ & $0.4^{[3]}$ & $0.3^{[3,4]}$ & $0.2^{[3]}$ & $0.1^{[6]}$ & $0.17^{[3]}$ & $0.02^{[5]}$ \\
C/1996 B2 (Hyakutake) & $0.8^{[7]}$ & $0.1^{[8]}$ & & & $0.1^{[6]}$ & $0.1^{[7]}$ & $0.01^{[9]}$ \\
C/2001 A2 (LINEAR) & $1.15^{[10]}$ & & & & $0.07^{[10]}$ & & \\
73P/SW3/B & $0.2-0.4^{[11]}$ & & & & $0.12-0.19^{[11]}$ & & \\
73P/ SW3/C & $0.16-0.3^{[11]}$ & & & & $0.08-0.14^{[11]}$ & & \\
9P/Tempel 1 before impact & $0.5^{[12]}$ & & & & & & \\
67P summer hemisphere & $0.67^{[13]}$ & $0.017^{[13]}$ & $0.004^{[13]}$ & $0.011^{[13]}$ & a) & $0.003^{[13]}$ & $0.0004^{[13]}$ \\
67P winter hemisphere & $1.75^{[13]}$ & $0.098^{[13]}$ & $0.0014^{[13]}$ & $0.041^{[13]}$ & a) & $0.024^{[13]}$ & $0.0013^{[13]}$ \\
\hline
\end{tabular}

Notes. This table is based on Table 1 in Bockelée-Morvan et al. (2004), on Table 4 in Mumma \& Charnley (2011) and on Table 3 in Radeva et al. (2013). References. [1] Eberhardt et al. (1994); [2] Feldman et al. (1987); [3] Bockelée-Morvan et al. (2000); [4] Lis et al. (1997b); [5] Despois et al. (2005); [6] Charnley \& Rodgers (2008); [7] Biver et al. (1999); [8] Woodney et al. (1997); [9] Weaver et al. (1996); [10] Biver et al. (2006) (values at 1.1 AU); [11] Biver et al. (2008); [12] Biver et al. (2007); [13] this work; comments: a) CS cannot be resolved from CO $_{2}$ by ROSINA.

In reality, however, the mass calibration relation is more complicated, especially when post-acceleration is applied. As a result of the non-symmetric electrical fields in the detection region, the ion beam is asymmetrically deflected. These dependencies on $M_{0}$ can be fitted using mass calibration measurements, in which an association can be made between the observed peaks (giving $p$ ) and the corresponding ion mass-percharge (giving $M$ ), and this for various commanded $M_{0}$ values. This has been done with the utmost caution, because in principle there is no unambiguous way to identify which ion is associated with a particular peak. However, as we show in this paper, many ions can indeed be identified as products of the ionization and/or fragmentation of cometary neutrals in the ion source. Nevertheless, the mass calibration fit remains an approximation because $p_{0}$ and $z$ do not only depend on $M_{0}$, but also on instrument parameters, in particular the magnet temperature. In practice, this means that the calibration relation has to be re-established for each measurement sequence.

\subsection{Determination of parent species and their abundances}

The measured signal is correlated with the density in the coma in several steps. First, for each peak of the selected mass spectra, the number of particles per time registered by the detector as a function of mass must be determined. From this, the parent species can be identified. Together with the instrument specific sensitivity factors and the fragmentation pattern for each species, the density of the species in the coma can be inferred. This last step then needs calibration data from the instrument. As each mass spectrometer has its own characteristics depending on geometry, ionization, and detector yield, the sensitivity, and the fragmentation pattern should be derived from measurements. Ideally, this should be done with the flight model before launch.

Despite the high-mass resolving power of DFMS, peaks in calibrated high-resolution spectra are often not completely separated, and peak-fitting is necessary to obtain individual ion currents. DFMS peak shapes can be modeled by two colocated Gaussians with a 9:1 intensity proportion and a 1:3 width ratio. Peak-fitting results in a determination of a peak area corresponding to each ion, which results in the total ion current and the total number of ions per second. As each mass is associated with an ion, we obtained a list of detected ions versus their total number per second.

The goal is then to infer which neutrals entered the instrument, and in what quantities. A complicated inversion has to be performed, typically based on the list of mass centroids versus number of particles, taking into account the fragmentation patterns of all likely neutrals, and possibly taking into account isotopic abundance ratios. Electron ionization (EI) of neutrals in the ion source can result in the formation of a singly charged atomic or molecular ion, but it can also produce multiply charged ions. In addition, a neutral molecule may break up into several fragment ions. The fragmentation pattern of a given neutral describes the identities and relative abundances of such product ions. The pattern depends on the design of the ion source and the electron energy used. In principle, this pattern has to be measured for DFMS for each species. For most relevant neutrals, this has been done using the instrument copy in the calibration facility. The list of the calibrated species can be found in Table 1 . For compounds that are poisonous, detrimental to the instrument, or otherwise impossible to measure, we rely on the literature. Generally, we used the National Institute of Standards and Technology (NIST) database for such compounds, even though the mass spectra in NIST have been acquired with an electron energy of $70 \mathrm{eV}$ compared to the $45 \mathrm{eV}$ electron beam in DFMS.

For this study, the parent species identification consisted of successively removing the fingerprint of the potential parents starting from the species with the highest mass. If all the parent species are identified, the remaining number of particles for each ion should be zero. The success of this identification strongly depends on the list of parent species selected and the correctness of their fragmentation patterns (measured with the copy of DFMS or coming from NIST). In our case, the parent list includes all the species that have previously been detected in cometary atmospheres and some new compounds that were tentatively identified in the data during the mass calibration. This step also enables calculating the correct number of particles for each parent species. When analyzing the spectrum of a mixture, some peaks (e.g., $\mathrm{CO}$ at $m / q=28$ ), are a combination of fragments (e.g., the ionization of $\mathrm{CO}_{2}$ leads to the $\mathrm{CO}$ fragment at $m / q=28$ ) and parent (e.g., parent $\mathrm{CO}$ in the coma). The 


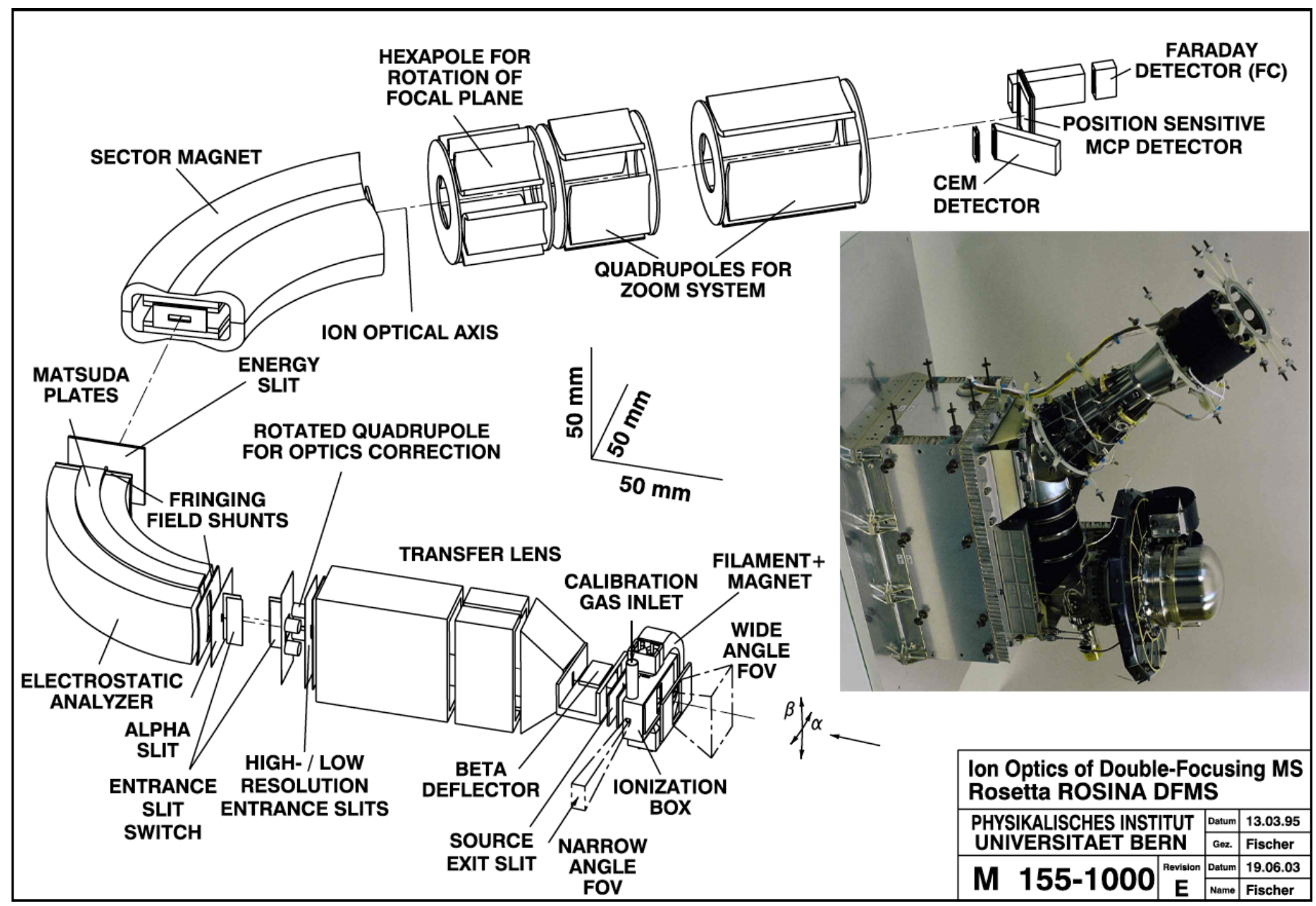

Fig. 2. DFMS ion optics: neutrals enter the source where they are ionized (bottom center) before they pass the electrostatic analyzer (left bottom), the magnet (top left), and then arrive at the detector (top right). Taken from Balsiger et al. (2007). The inset shows a picture of the DFMS sensor in the same orientation.

differentiation of isomers (molecules with the same molecular formula but where the atoms have a different arrangement in space) depends on the presence of considerably different fragmentation patterns between these isomers. More often than not, they cannot be distinguished from each other, and therefore the obtained result is not necessarily a unique solution.

The sensitivity of a mass spectrometer for a particular neutral gives the ratio of the detected total ion current to the neutral density in the instrument. This is the product of ionization cross section, instrument throughput, and detector yield. All these parameters are species dependent.

More specifically for DFMS, the relation between the ion current on the detector, $I_{i}$ (in $\mu \mathrm{A}$ ), sensitivity for gas $j, S_{\mathrm{j}}$ (in $\mathrm{cm}^{3}$ ), the electron emission current, $I_{\mathrm{em}}($ in $\mu \mathrm{A})$, the fragmentation pattern for ion species $i$ from gas $j$ (e.g., $\mathrm{O}^{+}$from $\left.\mathrm{CO}_{2}\right), \mathrm{Q}_{i j}$, and the neutral density, $n_{j}\left(\right.$ in $\mathrm{cm}^{-3}$ ) can be written as

$I_{i}=I_{\mathrm{em}} S_{j} Q_{i j} n_{j}$

or, if the parent species can readily be identified (e.g., $\mathrm{H}_{2} \mathrm{O}$ ),

$I_{\text {parent }}=I_{\mathrm{em}} S_{\text {parent }} n_{\text {parent }}$.

The sensitivity factors are key parameters to determine the abundances of the parent molecules identified in the coma. Calibration measurements with the ground model of DFMS were performed in the calibration chamber CASYMIR
(Westermann et al. 2001; Graf et al. 2004) to determine fragmentation pattern and sensitivity of the potential parent species in the coma of 67P. Nevertheless, it was clear from the beginning that not all the species could be analyzed for the reasons mentioned above.

To be able to determine the abundance of the species that cannot be (or have not been) studied in our calibration facility, we therefore determined from calibration measurements a function that links the mass-to-charge ratio to the sensitivity of the compounds. Table 1 lists the sensitivities and fragmentation patterns that were measured with the copy of DFMS and those calculated from this function. The uncertainties of the results depend on whether the compounds could be calibrated or not.

An example for a molecule that was calibrated in the calibration facility is given in Fig. $3 . \mathrm{H}_{2}{ }^{32} \mathrm{~S}$ is ionized and fragmented by the electron impact ion source: products can be found at mass $34 \mathrm{u} / \mathrm{e}$, where we see the parent $\mathrm{H}_{2}{ }^{32} \mathrm{~S}$ (and the heavy isotope ${ }^{34} \mathrm{~S}$, which is a fragment of $\mathrm{H}_{2}{ }^{34} \mathrm{~S}$, but also of other sulfurbearing species possibly present in the background of the vacuum chamber), at mass $33 \mathrm{u} / \mathrm{e}$ for $\mathrm{H}^{32} \mathrm{~S}$ (and the ${ }^{33} \mathrm{~S}$ isotope), and at mass 32 for the fragment ${ }^{32} \mathrm{~S}$. Comparison of the data from space then allowed us to determine the density of $\mathrm{H}_{2}{ }^{32} \mathrm{~S}$ at the entrance of DFMS. A mass spectrum of mass $34 \mathrm{u} / \mathrm{e}$ from space is shown in Fig. 4. Of course, in space as well as in the 


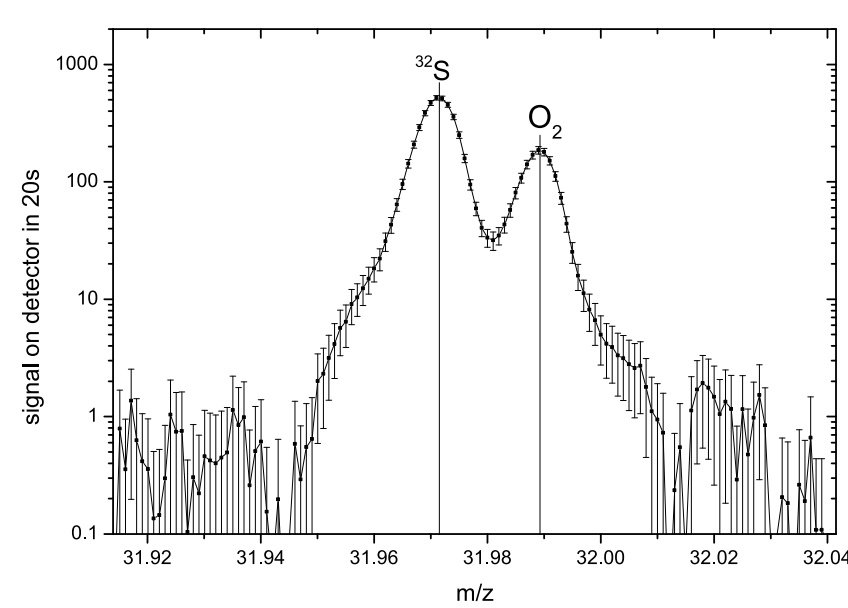

(a) Mass per charge $32 \mathrm{u} / \mathrm{e}$ spectrum

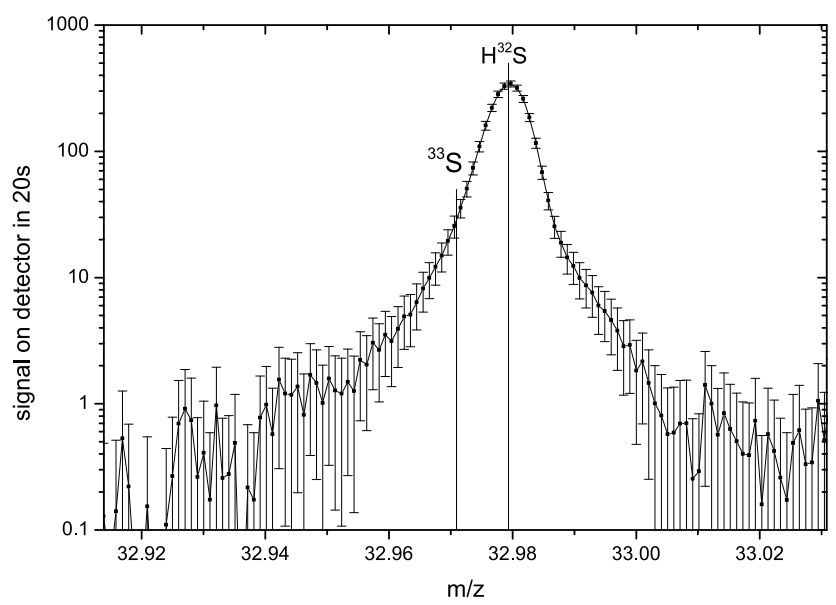

(b) Mass per charge $33 \mathrm{u} / \mathrm{e}$ spectrum

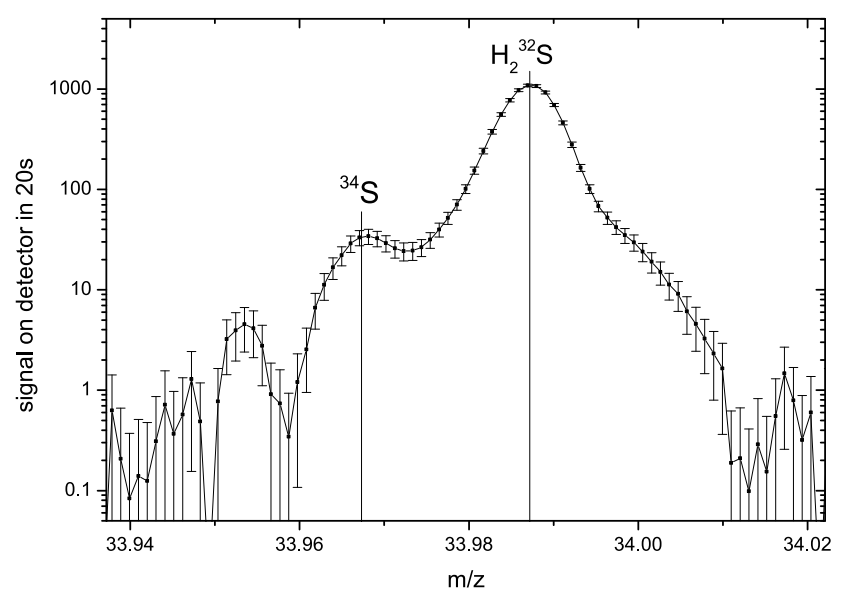

(c) Mass per charge $34 \mathrm{u} / \mathrm{e}$ spectrum

Fig. 3. Laboratory calibration measurement of $\mathrm{H}_{2} \mathrm{~S}$ with the corresponding sulfur isotopes and further contribution from vacuum-chamber background.

calibration facility, other molecules, atoms, or fragments may be on the same interger mass as shown in Fig. 4.

The curves in Fig. 4 were measured at different times. The black curve is background from the $\mathrm{S} / \mathrm{C}$, measured very far $\left(2.71 \times 10^{6} \mathrm{~km}\right)$ from the comet in April 2014, when the comet was at $4 \mathrm{AU}$ from the Sun. The $\mathrm{S} / \mathrm{C}$ is still outgassing, even after ten years in space (Schläppi et al. 2010). Some small signature

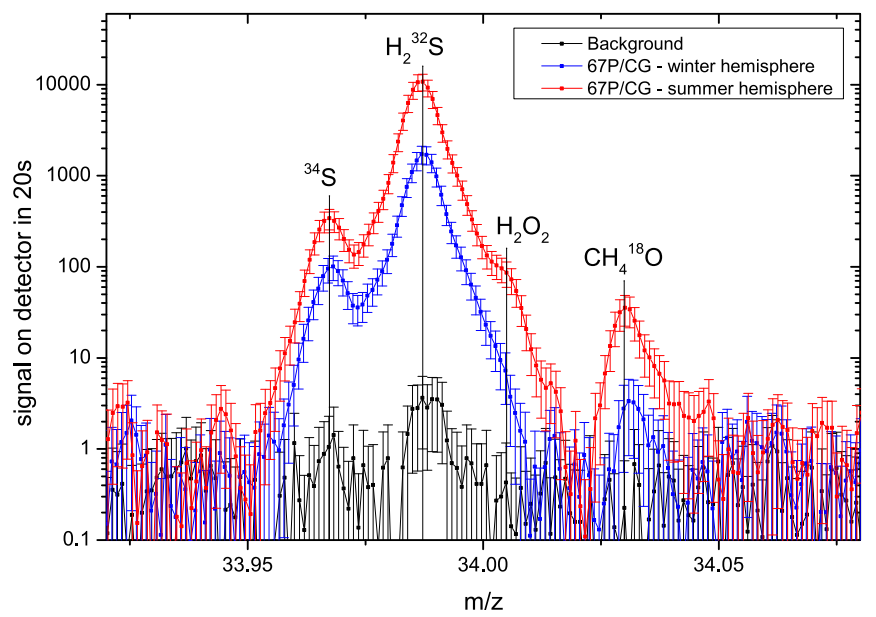

Fig. 4. In-flight mass per charge $34 \mathrm{u} / \mathrm{e}$ spectra showing spacecraft background (black) obtained in April 2014 before entering the coma of the comet and at a distance of $10 \mathrm{~km}$ from the center of the nucleus above the winter (blue) and the summer (red) hemisphere.

of $\mathrm{H}_{2}{ }^{32} \mathrm{~S}$ can be seen, which is probably due to conformal coating or electronics inside the $\mathrm{S} / \mathrm{C}$. The blue curve was measured at a distance of $10 \mathrm{~km}$ from the comet in a terminator orbit. The red curve was measured in the same orbit, but one day later.

Different sources contribute to the uncertainties of the derived densities: there is a statistical error given by the number of ions, which is mostly negligible unless the signal is very weak. Some uncertainty is due to the changing pixel gain and the overall detector gain. There is an error from curve fitting, especially if there are overlapping peaks. There is a contribution to the uncertainty from calibration, or in the case of compounds that could not be calibrated, from the estimate of instrument sensitivity and the fragmentation pattern.

For calibrated compounds, the uncertainties are generally around $20 \%$, mainly due to the pixel gain correction. For uncalibrated compounds, the uncertainties can be up to a factor of 2, principally explained by the error on the inferred sensitivity. To normalize the abundances, they are usually expressed relative to water. As DFMS measures one mass after the other, water is not measured simultaneously with the other species. The time difference can be up to $20 \mathrm{~min}$. This results in an uncertainty on the water abundance that can be partly compensated for by taking into account the COPS densities, which are transmitted every minute. However, the range given in Tables 2 to 5 is dominated not by instrument uncertainty, but by different outgassing according to Rosetta's position with respect to the comet and the Sun.

\section{Results and discussion}

We here limit ourselves to parent molecules that have previously been detected in comets. This limitation has a practical reason. To calibrate the instrument, the sensitivity and fragmentation pattern for each species have to be determined. The calibration prior to arrival at the comet was obviously focused on molecules previously known to be present in cometary comae (with some exceptions due to toxicity, corrosiveness, etc.). From our measurements at the comet, it is clear that other parent molecules are present. These molecules require laboratory calibration as well and will be presented in a later paper.

Rosetta arrived at the comet in early August 2014. Once it was within $100 \mathrm{~km}$ from the nucleus, the background from 
the $\mathrm{S} / \mathrm{C}$ became significantly lower than the signal from the coma (Bieler et al. 2015). The coma was already clearly water dominated, with strong contributions of $\mathrm{CO}$ and $\mathrm{CO}_{2}$, except over the winter hemisphere, where $\mathrm{CO}$ or even $\mathrm{CO}_{2}$ sometimes were the dominant species. It is clear not only from the relative abundances, but also from the different spatial distribution that $\mathrm{CO}$ must be predominantly a parent species and only a fraction ranging between a few percent up to $<40 \%$ originates from $\mathrm{CO}_{2}$ (Hässig et al. 2015).

Unexpectedly, a rich diversity of minor species such as sulfur-bearing compounds and hydrocarbons was found in the coma on arrival at the comet at $3.5 \mathrm{AU}$. The measurements in this paper were obtained at $3.15 \mathrm{AU}$, shortly before lander delivery, when the $\mathrm{S} / \mathrm{C}$ was on an orbit in the terminator plane at $10 \mathrm{~km}$ from the nucleus center. This corresponds to the time when ROSINA/COPS measured the highest gas densities in 2014. During these orbits, the $\mathrm{S} / \mathrm{C}$ flew over the winter as well as the summer hemisphere. The field of view (FOV) of DFMS is wide, so that particles outgassing from all areas facing the $\mathrm{S} / \mathrm{C}$ over the whole comet always contributed to the signal (Bieler et al. 2015). Over the winter hemisphere the S/C faced large poorly illuminated areas, whereas over the summer hemisphere most of the area facing the S/C was sunlit, see Figs. $1 \mathrm{~b}$ and c. We selected mass scans over the winter and summer hemisphere. The two scans were taken at approximately the same longitude, but at different latitudes (Fig. 1a). In both scans the S/C sees contributions from the summer and the winter hemispheres, but with different proportions.

Table 2 shows the relative abundance of the main species and hydrocarbons. CO varies between $2.7 \%$ and $20 \%$ relative to water. This is comparable to what has been measured in LP comets (cf. 13\% at comet 1P/Halley; Rubin et al. 2011), but higher than for JFCs. However, it has to be stated that these measurements were made at $3 \mathrm{AU}$, whereas most of the other measurements were obtained much closer to the Sun with higher water production. At the two locations $\mathrm{CO}_{2}$ varies between $2.5 \%$ and $80 \%$, which seems to be higher than at other comets such as Hale-Bopp and 103P/Hartley 2, again taking into account the heliocentric distance differences. Here the difference between summer and winter hemisphere is extremely large (cf. Hässig et al. 2015). Comparing these results with other instruments onboard the Rosetta $\mathrm{S} / \mathrm{C}$, we also observed much stronger $\mathrm{CO}_{2} / \mathrm{H}_{2} \mathrm{O}$ ratio variations than the Visible Infrared Thermal Imaging Spectrometer (VIRTIS). The $\mathrm{CO}_{2} / \mathrm{H}_{2} \mathrm{O}$ production rate ratio measured by VIRTIS-H is in the same order of magnitude, but ranges from 2 to 30\% (Bockelée-Morvan et al. 2015) compared to 2.5 to $80 \%$ in our study. In the regions with strong solar illumination, VIRTIS-H measured a mean value of between 3 and $6 \%$. This low value is compatible with our measurement $(2.5 \%)$ in the summer hemisphere. VIRTIS-H also derived an upper limit for the $\mathrm{CO} / \mathrm{H}_{2} \mathrm{O}$ column density ratio from specific $\mathrm{CO}_{2}$ measurements $\left(\mathrm{CO} / \mathrm{H}_{2} \mathrm{O}<1.8 \%\right)$. This value is somewhat lower than the value we obtained in the summer hemisphere $(2.7 \%)$. We conclude that our measurements roughly agree with $\mathrm{CO}_{2} / \mathrm{H}_{2} \mathrm{O}$ and $\mathrm{CO} / \mathrm{H}_{2} \mathrm{O}$ ratios determined by VIRTIS-H. Nevertheless, we note that the VIRTIS-H values have been obtained after averaging observations over a long and different time period (from 24 November 2014 to 24 January 2015). Moreover, as their data have been averaged, weaker variations in the column density ratios are expected. In addition, comparing local in situ measurments to line-of-sight integrated remote-sensing measurements can also lead to some differences.

For the hydrocarbons, while $\mathrm{CH}_{4}$ fits well with what has been obtained from other JFCs, $\mathrm{C}_{2} \mathrm{H}_{2}$ is rather abundant. This is even more pronounced for $\mathrm{C}_{2} \mathrm{H}_{6}$, which is up to $3.3 \%$ of the total number density on the winter hemisphere. This value is much higher than for any other comet. Moreover, the $\mathrm{C}_{2} \mathrm{H}_{6} / \mathrm{CH}_{4}$ ratio is higher than $1(\approx 2.5$ and $\approx 6$ in the summer and winter hemisphere, respectively). For comets C/2001 A2 (LINEAR) and C/1996 B2 (Hyakutake), for which the ethane relative abundance was also high, this ratio was roughly 1 (Magee-Sauer et al. 2008; Mumma et al. 1996). This high abundance of $\mathrm{C}_{2} \mathrm{H}_{6}$ relative to $\mathrm{CH}_{4}$ is interesting as it can provide insights into the chemistry occurring in the solar nebula. Mumma et al. (1996) have discussed the different scenarios: this ratio may indicate that acetylene, produced in the gas phase and condensed onto icy grains, has been hydrogenated in the interstellar dense cloud. It may also reflect the processes (irradiation) that the ices have undergone. The ion-molecule reactions to produce ethane are also mentioned. Nevertheless, these reactions are not favorable at low temperature, and currently we cannot exclude any of these possibilities.

On the other hand, it is not really clear which molecules contribute to the $\mathrm{C}_{2}$ chains used to apply the remote-sensing-based taxonomy of "carbon chain normal" or "depleted". Therefore, a classification in the same sense cannot be made from our data. Assuming that the contribution to $\mathrm{C}_{2}$ is mainly from $\mathrm{C}_{2} \mathrm{H}_{2}$ (see discussion in Weiler 2012), and that the contribution to $\mathrm{CN}$ is mainly from $\mathrm{HCN}$, we set $[\mathrm{HCN}]=[\mathrm{CN}]$ as well as $\left[\mathrm{C}_{2} \mathrm{H}_{2}\right]=$ $\left[\mathrm{C}_{2}\right]$ and $\left[\mathrm{H}_{2} \mathrm{O}\right]=[\mathrm{OH}]$ assuming that all parents are eventually photodissociated and contribute to the remotely observed column densities. From our data, the ratio of $\mathrm{CN} / \mathrm{OH}$ for $67 \mathrm{P}$ for the summer hemisphere is $\log ([\mathrm{CN}] /[\mathrm{OH}]) \sim-3$ and for $\log \left(\left[\mathrm{C}_{2}\right] /[\mathrm{OH}]\right) \sim-3.3$ (see Tables 2 and 4 ). The summer hemisphere has the highest outgassing and is most appropriate for comparison with remote sensing. These results would mean that $67 \mathrm{P}$ is carbon-chain depleted if we consider only the summer hemisphere. For the winter hemisphere, this completely changes, and $67 \mathrm{P}$ would be carbon-chain normal. This has to be taken with caution: the measurements are made at $3 \mathrm{AU}$ compared to less than $2 \mathrm{AU}$ for most other comets. It is reasonable to expect that the water production rate increases relative to more volatile species as the comet's heliocentric distances decreases (Biver et al. 2002). Once the comet will be bright enough for spectroscopic analysis from the ground and $\mathrm{C}_{2}, \mathrm{C}_{3}$, and $\mathrm{CN}$ can be measured, it should be possible to obtain a better picture of which parent molecules are responsible for the observed column densities of $\left[\mathrm{C}_{2}\right],\left[\mathrm{C}_{3}\right]$, and $[\mathrm{CN}]$.

Table 3 shows the relative abundance of the oxygenated species. Methanol falls well within the range of other JFCs. Luspay-Kuti et al. (2015) showed that methanol is more closely related to water than other more volatile species. The relative abundance of methanol therefore changes less than other more volatile species between summer and winter hemisphere. The same is true for formaldehyde. It was postulated that formaldehyde can also be produced from thermal degradation of grains and therefore has a distributed source (Meier et al. 1993). At $10 \mathrm{~km}$ from the coma, mostly direct sublimation from the nucleus surface is observed and, in addition, the temperatures are probably too low to allow breaking up of $\mathrm{H}_{2} \mathrm{CO}$ polymers at these distances from the Sun. It will be important to follow the evolution of $\mathrm{H}_{2} \mathrm{CO}$ with heliocentric and cometocentric distances to determine whether the grain production hypothesis holds true. All other oxygenated species are lower than observed at comets Hale-Bopp, Lemmon, or Lovejoy, the only three comets so far where they have been measured. No direct comparison can be made due to the different class of comets, and of course again due to heliocentric distance. Ethylene glycol 
has been detected at a level of $8 \times 10^{-6}$ compared to water. This is very low compared to concentrations in the three Oort cloud comets. Ethylene glycol has a high sublimation temperature, which may be the main reason for the very low abundance that is only observed over the summer hemisphere. But its presence nevertheless proves that Kuiper belt comets also contain complex organics. No clear peak of $\mathrm{NH}_{2} \mathrm{CHO}$ is present in our mass spectra, but it might be hidden by another peak present at this mass. Therefore, we derived an upper limit for this compound.

Table 4 shows the relative abundance of the nitrogen-bearing species. HCN, and HNCO are probably typical for comets in general, as the values do not differ very much between the different classes. Little $\mathrm{NH}_{3}$ and $\mathrm{CH}_{3} \mathrm{CN}$ have been observed. No well-resolved cyanoacethylene peak at $m / q=51$ is found. We here also calculated an upper limit. Taken together with the $\mathrm{N}_{2}$ value measured by Rubin et al. (2015), our measurements of 67P confirm that cometary volatiles are depleted in nitrogen.

Table 5 shows the relative abundance of the sulfur-bearing species. The abundance of $\mathrm{H}_{2} \mathrm{~S}$ in $67 \mathrm{P}$ is comparable to the values measured in LPCs, but 67P is depleted in all other sulfurbearing species. Here we have to be cautious: except for $\mathrm{H}_{2} \mathrm{~S}$, no other sulfur-bearing species has been calibrated due to their poisonous or corrosive nature. Error bars may therefore be high. Except for $\mathrm{H}_{2} \mathrm{~S}$ and $\mathrm{CS}$ in comet 73P/SW3, no sulfur-bearing species have ever been measured in JFCs, which precludes a direct comparison. The higher relative abundance value of SO in the summer hemisphere compared to the winter hemisphere is really surprising. An explanation might be that SO might be a fragment of molecules with even higher masses (other than $\mathrm{SO}_{2}$ ). No such molecules were detected in our data, but it might just be that their abundances are not high enough. To explain this ratio, however, this also means that these potentially unidentified parents are stronger in the summer than in the winter hemisphere.

The ratio of water production for the two measurement periods is approximately $\mathrm{H}_{2} \mathrm{O}_{\text {summer }} / \mathrm{H}_{2} \mathrm{O}_{\text {winter }}=16$. For $\mathrm{CO}_{2}$, this ratio is much lower. The absolute number of neutral detected with DFMS is even higher in the winter than in the summer hemisphere. This means that its their absolute production rate is higher over the winter than over the summer hemisphere. We calculated the summer-to-winter hemisphere abundance ratio for $\mathrm{CO}_{2}$ and found that it is around 0.5. This cannot be explained by temperature differences between summer and winter hemisphere. For $\mathrm{CO}, \mathrm{CH}_{4}, \mathrm{C}_{2} \mathrm{H}_{2}, \mathrm{C}_{2} \mathrm{H}_{6}, \mathrm{HCN}, \mathrm{CS}_{2}$, and OCS, the summer-to-winter hemisphere abundance ratio is also lower than that of water. It is between 1 and 4. (see Luspay-Kuti et al. 2015 and Hässig et al. 2015). These large differences may reflect the evolution of the cometary surface: the northern hemisphere was processed over the last orbit, during which it was the summer hemisphere for $5.5 \mathrm{yr}$ and lost most of its highly volatiles, but relatively little $(<1 \mathrm{~m})$ of its surface layer. This surface layer may therefore be depleted in volatiles and/or closed off for sublimation from deeper layers. A chemically differentiated nucleus such as this has been modeled by Flammer et al. (1998) for HaleBopp, where they assumed an evolution of a dust layer on top followed by a devolatized ice-dust layer underneath that partly inhibited the sublimation of volatiles from deeper layers. For $67 \mathrm{P}$ this is further complicated by the rotation axis and the irregular shape that leads to pronounced seasonal effects, where the thickness of these layers may vary considerably between hemispheres and locations on the nucleus. The southern hemisphere, which is the winter hemisphere during the time of the reported observations, has become sunlit in May 2015 and then experienced a short, but intense summer, during which time it eroded by a few meters (Keller et al. 2015) and probably again exposed layers containing many highly volatile species. This can be studied in the next few months around perihelion and might then shed light on how volatiles are trapped in the cometary ice. This is needed to be able to connect our measurements to the bulk composition and also to ground-based observations.

\section{Conclusions}

The ROSINA instrument has detected many volatile species in the cometary coma at 3 AU. The relative abundances differ significantly between summer and winter hemispheres, most probably due to the surface evolution of the comet. In general, the mean values obtained are comparable with measurements from other comets with a few notable exceptions: $\mathrm{CO}$ and $\mathrm{CO}_{2}$ are very abundant compared to those measured in JFCs. Heavy oxygen-bearing molecules are depleted, but most probably due to their high sublimation temperatures. Ethane is very abundant on the southern (winter) hemisphere. $\mathrm{N}$-bearing molecules are typical, but together with the low measured $N_{2}$ (Rubin et al. 2015), nitrogen is clearly depleted.

From the ROSINA measurements we cannot conclude that this comet is carbon-chain normal or depleted because it is not clear which parent molecules contribute to the measured column densities from remote sensing. Because relative abundances are very different for the two hemispheres, it is hard to say at this time which values represent the bulk abundance. It will be important to monitor the changes during the equinox in May 2015 as well as during perihelion. It will also be important to have combined measurement campaigns with remote-sensing instruments to find the parents of $\left[\mathrm{C}_{2}\right],\left[\mathrm{C}_{3}\right]$, and $[\mathrm{CN}]$ and to be able to compare data from the different regions above the nucleus with what is seen from Earth.

Acknowledgements. The authors thank the following institutions and agencies, which supported this work: work at the University of Bern was funded by the State of Bern, the Swiss National Science Foundation, and the European Space Agency PRODEX Program. Work at the Southwest Research Institute was supported by subcontract no. 1496541 from the Jet Propulsion Laboratory (JPL). Work at BIRA-IASB was supported by the Belgian Science Policy Office via PRODEX/ROSINA PEA 90020. This work was supported by CNES grants at LATMOS and LPC2E. Work at the University of Michigan was funded by NASA under contract JPL-1266313. The results from ROSINA would not be possible without the work of the many engineers, technicians, and scientists involved in the mission, in the Rosetta spacecraft, and in the ROSINA instrument team over the past $20 \mathrm{yr}$, whose contributions are gratefully acknowledged. We here acknowledge the work of the whole European Space Agency (ESA) Rosetta team. Rosetta is an ESA mission with contributions from its member states and NASA. All ROSINA data are available on request until they are released to the Planetary Science Archive of ESA and the Planetary Data System archive of NASA.

\section{References}

A'Hearn, M. F., Millis, R. C., Schleicher, D. O., Osip, D. J., \& Birch, P. V. 1995, Icarus, 118, 223

A'Hearn, M. F., Belton, M. J., Delamere, W. A., et al. 2011, Science, 332, 1396 Altwegg, K., Balsiger, H., \& Geiss, J. 1994, A\&A, 290, 318

Balsiger, H., Altwegg, K., Bochsler, P., et al. 2007, Space Sci. Rev., 128, 745 Berthelier, J.-J., Illiano, J.-M., Nevejans, D., et al. 2002, Int. J. Mass Spectr., 215, 89

Bieler, A., Altwegg, K., Balsiger, H., et al. 2015, A\&A, 583, A7

Bird, M., Janardhan, P., Wilson, T., et al. 1997, Earth, Moon, Planets, 78, 21

Biver, N., Bockelée-Morvan, D., Crovisier, J., et al. 1999, AJ, 118, 1850

Biver, N., Bockelee-Morvan, D., Colom, P., et al. 2002, Earth, Moon, Planets, 90,5

Biver, N., Bockelée-Morvan, D., Boissier, J., et al. 2007, Icarus, 187, 253

Biver, N., Bockelée-Morvan, D., Crovisier, J., et al. 2006, A\&A, 449, 1255

Biver, N., Bockelée-Morvan, D., Crovisier, J., et al. 2008, in Asteroids, Comets, Meteors 2008, LPI Contribution, 1405, 8149 
Biver, N., Bockelée-Morvan, D., Debout, V., et al. 2014, A\&A, 566, L5 Bockelée-Morvan, D. 1997, in Molecules in Astrophysics: Probes and Processes, IAU Symp., 178, 219

Bockelée-Morvan, D., Brooke, T. Y., \& Crovisier, J. 1995, Icarus, 116, 18

Bockelée-Morvan, D., Lis, D., Wink, J., et al. 2000, A\&A, 353, 1101

Bockelée-Morvan, D., Crovisier, J., Mumma, M. J., \& Weaver, H. A. 2004, in The composition of cometary volatiles, ed. G. W. Kronk (University of Arizona Press), 391

Bockelée-Morvan, D., Debout, V., Erard, S., et al. 2015, A\&A, 583, A6

Brooke, T., Tokunaga, A., Weaver, H., et al. 1996, Nature, 383, 606

Charnley, S., \& Rodgers, S. 2008, Space Sci. Rev., 138, 59

Cochran, A., Barker, E., \& Gray, C. 2012, Icarus, 218, 144

Colangeli, L., Epifani, E., Brucato, J., et al. 1999, A\&A, 343, L87

Combes, M., Crovisier, J., Encrenaz, T., Moroz, V. I., \& Bibring, J.-P. 1988, Icarus, 76, 404

Combi, M. R., \& Delsemme, A. H. 1980, ApJ, 237, 641

Combi, M. R., \& Fink, U. 1997, ApJ, 484, 879

Crovisier, J., Leech, K., Bockelee-Morvan, D., et al. 1997, Science, 275, 1904

Crovisier, J., Bockelée-Morvan, D., Biver, N., et al. 2004, A\&A, 418, L35

Crovisier, J., Biver, N., Bockelée-Morvan, D., \& Colom, P. 2009, Planet. Space Sci., 57, 1162

Dello Russo, N., Mumma, M. J., DiSanti, M. A., Magee-Sauer, K., \& Novak, R. 2001, Icarus, 153, 162

Dello Russo, N., Vervack, R. J., Weaver, H. A., et al. 2007, Nature, 448, 172 Dello Russo, N., Vervack, Jr., R. J., Weaver, H. A., et al. 2008, ApJ, 680, 793

Dello Russo, N., Vervack, Jr., R. J., Weaver, H. A., et al. 2009, ApJ, 703, 187

Dello Russo, N., Vervack, Jr., R. J., Lisse, C. M., et al. 2011, ApJ, 734, L8

Despois, D., Crovisier, J., Bockelee-Morvan, D., Gerard, E., \& Schraml, J. 1986, A\&A, 160, L11

Despois, D., Biver, N., Bockelée-Morvan, D., \& Crovisier, J. 2005, IAU Proc., 1,469

DiSanti, M. A., Mumma, M. J., Dello Russo, N., \& Magee-Sauer, K. 2001, Icarus, 153, 361

DiSanti, M. A., Mumma, M. J., Dello Russo, N., Magee-Sauer, K., \& Griep, D. M. 2003, J. Geophys. Res.: Planets, 108

DiSanti, M. A., Anderson, W. M., Villanueva, G. L., et al. 2007a, ApJ, 661, L101

DiSanti, M. A., Villanueva, G. L., Bonev, B. P., et al. 2007b, Icarus, 187, 240

Eberhardt, P. 1999, Space Sci. Rev., 90, 45

Eberhardt, P., Meier, R., Krankowsky, D., \& Hodges, R. R. 1994, A\&A, 288, 315

Feldman, P. D., Festou, M. C., Ahearn, M. F., et al. 1987, A\&A, 187, 325

Fink, U. 2009, Icarus, 201, 311

Fink, U., Combi, M. R., \& Disanti, M. A. 1991, ApJ, 383, 356

Fischer, G., Geith, J., Klapotke, T. M., \& Krumm, B. 2002, Z. Naturforschung B, 57,19

Flammer, K. R., Mendis, D. A., \& Houpis, H. L. F. 1998, ApJ, 494, 822

Fray, N., Bénilan, Y., Cottin, H., Gazeau, M.-C., \& Crovisier, J. 2005, Planet. Space Sci., 53, 1243

Gibb, E. L., Mumma, M. J., Dello Russo, N., DiSanti, M. A., \& Magee-Sauer, K. 2003, Icarus, 165, 391

Gibb, E. L., DiSanti, M. A., Magee-Sauer, K., et al. 2007, Icarus, 188, 224

Graf, S., Altwegg, K., Balsiger, H., et al. 2004, J. Geophys. Res.: Planets, 109

Hässig, M., Altwegg, K., Balsiger, H., et al. 2015, Science, 347, 0276

Helbert, J., Rauer, H., Boice, D. C., \& Huebner, W. F. 2005, A\&A, 442, 1107
Irvine, W. M., Bockelee-Morvan, D., Lis, D. C., et al. 1996, Nature, 383, 418

Kawakita, H., Kobayashi, H., Russo, N. D., et al. 2013, Icarus, 222, 723

Keller, H. U., Mottola, S., Davidsson, B., et al. 2015, A\&A, 583, A34

Krankowsky, D., Lammerzahl, P., Herrwerth, I., et al. 1986, Nature, 321, 326

Langland-Shula, L. E., \& Smith, G. H. 2011, Icarus, 213, 280

Lis, D., Keene, J., Young, K., et al. 1997a, Icarus, 130, 355

Lis, D., Mehringer, D., Benford, D., et al. 1997b, Earth, Moon, Planets, 78, 13

Lis, D. C., Bockelée-Morvan, D., Boissier, J., et al. 2008, ApJ, 675, 931

Luspay-Kuti, A., Hässig, M., Fuselier, S. A., et al. 2015, A\&A, 583, A4

Magee-Sauer, K., Mumma, M. J., DiSanti, M. A., Dello Russo, N., \& Rettig, T. W. 1999, Icarus, 142, 498

Magee-Sauer, K., Mumma, M. J., DiSanti, M. A., \& Dello Russo, N. 2002, J. Geophys. Res.: Planets, 107, 6

Magee-Sauer, K., Mumma, M. J., DiSanti, M. A., et al. 2008, Icarus, 194, 347

McPhate, J., Feldman, P., Weaver, H., et al. 1996, BAAS, 28, 1093

Meier, R., Eberhardt, P., Krankowsky, D., \& Hodges, R. R. 1993, A\&A, 277, 677

Meier, R., Eberhardt, P., Krankowsky, D., \& Hodges, R. 1994, A\&A, 287, 268

Mumma, M. J., \& Charnley, S. B. 2011, ARA\&A, 49, 471

Mumma, M. J., \& Reuter, D. C. 1989, ApJ, 344, 940

Mumma, M. J., DiSanti, M. A., Dello Russo, N., Fomenkova, M., et al. 1996, Science, 272, 1310

Mumma, M. J., DiSanti, M. A., Russo, N. D., Magee-Sauer, K., \& Rettig, T. W. 2000, ApJ, 531, L155

Mumma, M., DiSanti, M., Dello Russo, N., et al. 2003, Adv. Space Res., 31, 2563

Mumma, M. J., DiSanti, M. A., Magee-Sauer, K., et al. 2005, Science, 310, 270

Mumma, M. J., Bonev, B. P., Villanueva, G. L., et al. 2011, ApJ, 734, L7

Nevejans, D., Neefs, E., Kavadias, S., Merken, P., \& Van Hoof, C. 2002, Int. J. Mass Spectrometry, 215, 77

Ootsubo, T., Kawakita, H., Hamada, S., et al. 2012, ApJ, 752, 15

Paganini, L., DiSanti, M. A., Mumma, M. J., et al. 2014, AJ, 147, 15

Palmer, P., Wootten, A., Butler, B., et al. 1996, in BAAS, 28, 927

Radeva, Y. L., Mumma, M. J., Villanueva, G. L., et al. 2013, Icarus, 223, 298

Rubin, M., Tenishev, V. M., Combi, M. R., et al. 2011, Icarus, 213, 655

Rubin, M., Altwegg, K., Balsiger, H., et al. 2015, Science, 348, 232

Schläppi, B., Altwegg, K., Balsiger, H., et al. 2010, J. Geophys. Res.: Space Physics, 115

Schloerb, F. P., Kinzel, W. M., Swade, D. A., \& Irvine, W. M. 1987, A\&A, 187, 475

Villanueva, G. L., Bonev, B. P., Mumma, M. J., et al. 2006, ApJ, 650, L87

Weaver, H. A., Feldman, P. D., McPhate, J. B., et al. 1994, ApJ, 422, 374

Weaver, H., Feldman, P., A'Hearn, M., et al. 1996, IAU Circ., 6374, 1

Weaver, H., Chin, G., Bockelée-Morvan, D., et al. 1999, Icarus, 142, 482

Weaver, H. A., Feldman, P. D., A’Hearn, M. F., Dello Russo, N., \& Stern, S. A 2011, ApJ, 734, L5

Weiler, M. 2012, A\&A, 538, A149

Westermann, C. B., Luithardt, W., Kopp, E., et al. 2001, Measurement Science, 12,1594

Woodney, L., McMullin, J., \& A'Hearn, M. 1997, Planet. Space Sci., 45, 717

Woodney, L., A'Hearn, M., Schleicher, D. G., et al. 2002, Icarus, 157, 193 